\title{
Acceso venoso difícil en pediatría
}

\section{Difficult venous access in pediatrics}

\author{
Evelyn Borchert B. MD. ${ }^{1}$, Héctor J. Lacassie MD. ${ }^{1}{ }^{*}$, Mario Concha P. MD. ${ }^{1}$, Marcos Rattalino F. MD. ${ }^{1}$, Guillermo Lema F. MD. ${ }^{1}$ \\ 1 División de Anestesiología Facultad de Medicina Pontificia Universidad Católica de Chile.
}

Fuente de financiamiento del estudio: Fondos departamentales.

Los autores declaran no tener conflicto de interés.

Fecha de recepción: 18 de abril de 2021 / Fecha de aceptación: 03 de mayo de 2021

\begin{abstract}
Background: Cannulation of a peripheral venous access is a routine procedure in pediatric patients admitted to the hospital. $50 \%$ of the time cannulation on the first attempt is not feasible, so it is necessary to repeat the puncture, resulting in a complex and frustrating procedure. Half of the children admitted to the hospital have a difficult venous access (DIVA). Objective: To carry out a review, which provides information about DIVA in pediatrics, how to evaluate and proceed in these patients. Design: DIVA Score considers vein palpability, vein visibility, age and history of prematurity. The score ranges from 0 to 10 points. Values greater than 4 are associated with difficult venous access. There are associated risk factors: obesity, musculoskeletal malformations, chemotherapy treatment, diabetes mellitus, patients on dialysis, limb edema, moderate to severe dehydration, history of difficult venous access, anxiety of the patient and/or parents report that the child is less likely to cooperate. When making the decision to establish a venous access, it should be evaluated whether it is an emergency or not, the characteristics of the medications and infusions, the time of therapy and the anatomical sites for puncture. Ultrasound and transillumination techniques decrease the time to obtain a venous access and increase the success rate on the first attempt. Conclusion: The decision to obtain a venous access must take into account the criteria and risk factors for DIVA. The most recognized scale is the DIVA Score.
\end{abstract}

Key words: Phlebotomy; veins; pediatrics; ultrasonography.

\section{RESUMEN}

Introducción: La canulación de un acceso venoso periférico es un procedimiento rutinario en los pacientes pediátricos que ingresan al hospital. 50\% de las veces la canulación al primer intento es frustra, por lo que es necesario repetir la punción resultando el procedimiento complejo y frustrante. La mitad de los niños que ingresa al hospital presentan un acceso venoso difícil (DIVA, sigla derivada de "difficult intravenous access"). Objetivo: Realizar una revisión, que entrega información acerca de DIVA en pediatría, cómo evaluar y proceder en estos pacientes. Desarrollo: DIVA Score considera palpabilidad y visibilidad venosa, edad y antecedente de prematurez. El puntaje va desde 0 a 10 puntos. Valores mayores a 4 se asocian a acceso venoso difícil. Existen factores de riesgo asociados: obesidad, malformaciones osteomusculares, tratamiento con quimioterapia, diabetes mellitus, pacientes en diálisis, edema de extremidades, deshidratación moderada a severa, historia de acceso venoso difícil, ansiedad del paciente y/o padres que refieren que el niño es poco probable que coopere. Al tomar la decisión de establecer un acceso venoso se debe evaluar si es urgencia o no, las características de los medicamentos e infusiones, tiempo de terapia y los sitios anatómicos para punción. La ultrasonografía y las técnicas de transiluminación disminuyen el tiempo de obtención del acceso venoso e incrementan la tasa de éxito en el primer intento. Conclusión: En la decisión de obtener un acceso venoso se deben tener en cuenta los criterios y factores de riesgo de acceso venoso difícil. La escala más reconocida es el DIVA Score.

Palabras clave: Flebotomía; venas; pediatría; ultrasonografía. 


\section{Definición y reconocimiento del problema}

a obtención de un acceso venoso periférico es un procedimiento de rutina que se realiza en los pacientes pediátricos que ingresan al hospital con el fin de administrar un tratamiento médico. Sin embargo, 50\% de las veces la canulación de la vía venosa al primer intento es frustra, por lo que es necesario repetir la punción[1],[2], siendo necesario muchas veces el uso de punciones múltiples, resultando el procedimiento complejo, frustrante y todo un desafío para el personal médico y de enfermería[3].

Se estima que la mitad de los niños que ingresan al hospital (sobre todo menores de 3 años) presentan un acceso venoso difícil (DIVA, sigla derivada de "difficult intravenous access"), debido a factores intrínsecos y extrínsecos, dentro de los cuales destacan: edad, tejido adiposo, prematuridad, enfermedad e injuria (deshidratación y/o sepsis) y la experiencia del clínico que realiza el procedimiento (experiencia, habilidades y confianza[1]-[3].

Se considera DIVA a todo procedimiento en el que se requieren más de tres punciones para lograr la canulación venosa exitosa o cuando el procedimiento tarda más de 30 minutos. En promedio los niños reciben dos punciones, mientras que niños con DIVA pueden recibir hasta nueve punciones[3],[4]. Se ha establecido que los niños y sus padres manifiestan que la obtención de una vía venosa es el evento más traumático durante la hospitalización[1]-[4]. Por otra parte, los repetidos intentos de canulación agotan las venas utilizables para el futuro, obstruyendo el acceso vascular a largo plazo e incluso de por vida.

El acceso venoso difícil se asocia a retraso en el diagnóstico, inicio tardío de tratamientos, incremento en las complicaciones como infiltración de los tejidos blandos, extravasación de medicamentos, perforación vascular e infección y, por ende, aumento innecesario del consumo de los recursos de salud. Lo anterior puede verse relacionado con aumento de la morbilidad, mortalidad y estadía hospitalaria[1]-[4].

Varias revisiones sistemáticas con metaanálisis han demostrado que el uso de ultrasonido incrementa el éxito de las punciones, por lo que se recomienda su uso en pacientes en quienes se predice un acceso venoso difícil, al igual que las técnicas de transiluminación. Ambas técnicas disminuyen el tiempo de obtención del acceso venoso e incrementan la tasa de éxito del procedimiento en el primer intento[4]-[6].

La siguiente revisión tiene como objetivos evaluar los hallazgos o indicadores que nos advierten de un posible DIVA; de acuerdo con esto, la forma de proceder y qué técnicas pueden ser útiles para facilitar el procedimiento. Finalmente, describir un algoritmo de trabajo ajustado a nuestra realidad cuando se identifique un DIVA.

\section{Desarrollo}

\section{Predicción de acceso venoso difícil}

Existe una tabla con criterios para definir un acceso venoso difícil, denominada DIVA Score, que fue validada en una población de 46.000 pacientes de 0 a 21 años. Las variables se muestran en la Tabla 1[3],[4].

El puntaje va desde 0 a un máximo de 10 puntos. Se ha de- mostrado que cuando se obtienen valores mayores a 4 se suelen requerir varias punciones para la obtención de un adecuado acceso venoso, con una especificidad de $92,7 \%$, sensibilidad de $21,4 \%$ y un valor predictivo positivo de $49,5 \%$. En algunos estudios se ha encontrado que cuando DIVA Score es de 4 se presenta $54 \%$ de fallos en el primer intento de canulación y en algunas ocasiones se requiere de intentos por personal más experimentado. Con una puntuación de 8 la probabilidad de canular al primer intento es mínima, siendo necesario recurrir al uso de ayuda para establecer el acceso venoso periférico o a otro tipo de acceso (catéter venoso central)[3],[4].

Desde su inicio, el DIVA Score ha sido modificado en una versión de tres ítems (DIVA 3: vena palpable, vena visible y edad[7], validada con la misma especificidad $(93,5 \%)$ y sensibilidad (22,3\%)[3] y redefinida también para pacientes adultos[8], que posee la misma capacidad para discriminar un acceso venoso difícil en cuanto a la sensibilidad y especificidad para la obtención de una vía venosa al primer intento.

\section{Factores de riesgo}

Además de los predictores indicados en el DIVA Score se han encontrado otras características de los pacientes que se asocian a acceso venoso difícil[1]-[4]:

- Obesidad: los niños obesos requieren más de una punción para ser canulados.

- Malformaciones osteomusculares de las extremidades.

- Edema de extremidades debido a tratamientos farmacológicos o quirúrgicos.

- Uso previo de medicamentos que lesionen los trayectos venosos.

- Quimioterapia.

- Diálisis.

- Diabetes mellitus.

- Deshidratación moderada a grave.

- Historia previa de punciones múltiples.

- Ansiedad por parte del paciente.

En un estudio reciente[3] se identificaron factores de riesgo independientes para acceso venoso difícil en pacientes pediátricos con DIVA Score o DIVA 3 menor a 4, entre los que destacan: predicción por enfermera capacitada (entrenada y con experiencia), deshidratación moderada a grave, historia previa de acceso venoso difícil, predicción de los padres de que el niño no

\begin{tabular}{lll}
\hline \multicolumn{3}{c}{ Tabla 1. Cálculo del DIVA Score } \\
\hline Variable & Opciones & Puntos \\
Vena visible después de torniquete & Visible & 0 \\
& No visible & 2 \\
Vena palpable después de torniquete & Palpable & 0 \\
& No palpable & 2 \\
Edad & $\geq 3$ años & 0 \\
& $1-2$ años & 1 \\
& $<1$ año & 3 \\
Antecedente de prematurez edad gestacional & No & 0 \\
$<38$ sem) & Sí & 3 \\
\hline
\end{tabular}


cooperaría con el procedimiento. En pacientes con DIVA Score o DIVA 3 mayor o igual a 4 se encontró que sólo la predicción por parte de la enfermera (entrenada y con experiencia) de un acceso venoso difícil constituye un factor de riesgo independiente.

Además de los factores descritos previamente, el acceso venoso difícil se puede ver influenciado por otros factores como son: el manejo del dolor y de la ansiedad del niño, las características ambientales y espaciales de la locación donde se realiza la punción, las estrategias que el operador pueda plantear desde un inicio para mejorar la visualización y la palpación de las estructuras venosas.

\section{Consideraciones para obtención de una vía venosa}

Para poder definir un lugar de punción se deben establecer sitios donde se logren ver y palpar las estructuras venosas. En algunas situaciones, sin embargo, deberemos puncionar sin ver o tocar la vena, para lo cual es preciso saber por donde van las venas, como sucede con alguna frecuencia con la vena safena interna. Esta se ubica por delante del maléolo interno ascendiendo ligeramente en diagonal (Figura 1). Si esperamos siempre tocarla o verla, perderemos una oportunidad de obtener una vena de buen calibre, extremadamente constante en su ubicación y que con alguna práctica no resulta tan difícil de canular. La experiencia del anestesiólogo resulta fundamental para buscar estas venas.

En el momento de tomar la decisión de establecer un acceso venoso se deben tener en cuenta los siguientes aspectos:

- Estado del paciente: definir urgencia o no urgencia. Si el procedimiento es de urgencia o si la patología requiere hospitalización posoperatoria prolongada se debe considerar la obtención de accesos venosos periféricos en sitios donde por localización logren permanecer funcionales por mayor tiempo. En estos casos se recomienda canular zonas como el dorso de la mano y el antebrazo, evitando puncionar los pliegues[3],[4],[9].

- Características de los medicamentos e infusiones que se van a administrar.

- Tiempo de terapia que requiere el paciente.

- Sitios anatómicos para punción venosa: a pesar de que en pediatría podemos no palpar o visualizar una vena, es muy importante conocer los sitios anatómicos en los que se encuentran:

- Vena safena interna: por delante del maléolo interno (Figura 1).

- Venas superficiales en la cara anterior de la muñeca.

- Venas de la fosa cubital: braquial, cefálica y basílica.

- Vena radial superficial en la cara lateral de la muñeca.

- Vena superficial del pulgar.

- Borde cubital del antebrazo.

- Vena yugular externa.

- Vena superficial en la cara interna de la tibia.

- Técnicas de apoyo para la obtención de una vía venosa: ecografía y transiluminación.

Ultrasonografía: En la última década el empleo del ultrasonido ha aumentado el éxito de los accesos venosos periféricos, como también la colocación de catéteres venosos

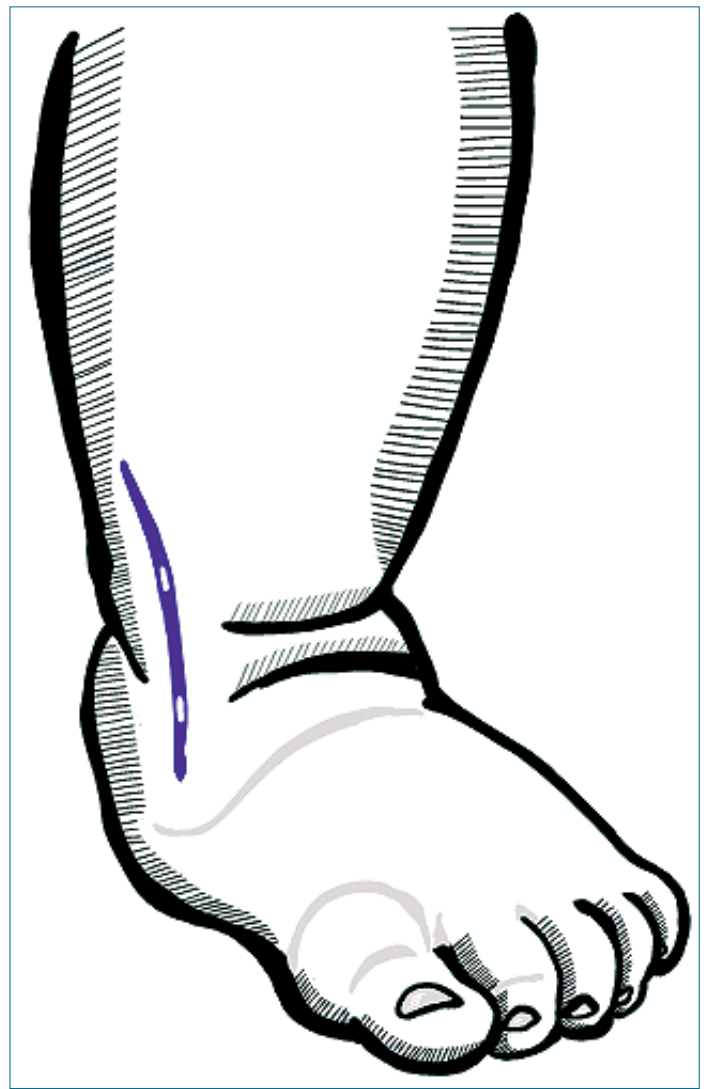

Figura 1. Vena safena interna izquierda.

centrales en situaciones de emergencia y en pacientes pediátricos.

La punción de venas periféricas se realiza con un transductor lineal de frecuencias entre 5 y $15 \mathrm{MHz}$, con una profundidad en la imagen de 2 a $5 \mathrm{~cm}$. Los avances tecnológicos en ultrasonido, la mayor definición y resolución de los transductores y la ecografía doppler color permiten visualizar imágenes en tiempo real y de mejor definición para la ubicación de los accesos venosos periféricos, por lo que en pacientes con DIVA se logra la canulación 6-7 veces más rápida que cuando no se usa ecografía, con un teflón de mayor calibre (22G en vez de $24 \mathrm{G}$ ), menor número de punciones ( 1 versus 2,5 ) y mayor tasa de éxito de canulación al primer intento. Así mismo, el uso de esta tecnología se asocia a una disminución del riesgo de punción arterial[4],[6],[10].

Se debe contemplar este recurso desde la primera punción en niños en quienes se considera que existen factores de riesgo de punción venosa difícil o en pacientes en quienes tras el segundo intento no se logra canular la vena, evitando así traumatismos adicionales e innecesarios y permitiendo disminuir los efectos negativos que conllevan las múltiples punciones, tanto a los niños como a sus familiares[3],[4],[6]. Transiluminación: Es una técnica que se ha realizado desde hace mucho tiempo para aumentar el éxito en la canulación venosa.

Se ha demostrado que el uso de transiluminación con LED permite disminuir el tiempo de obtención del acceso venoso y también incrementa la tasa de éxito en el primer intento[4]. 


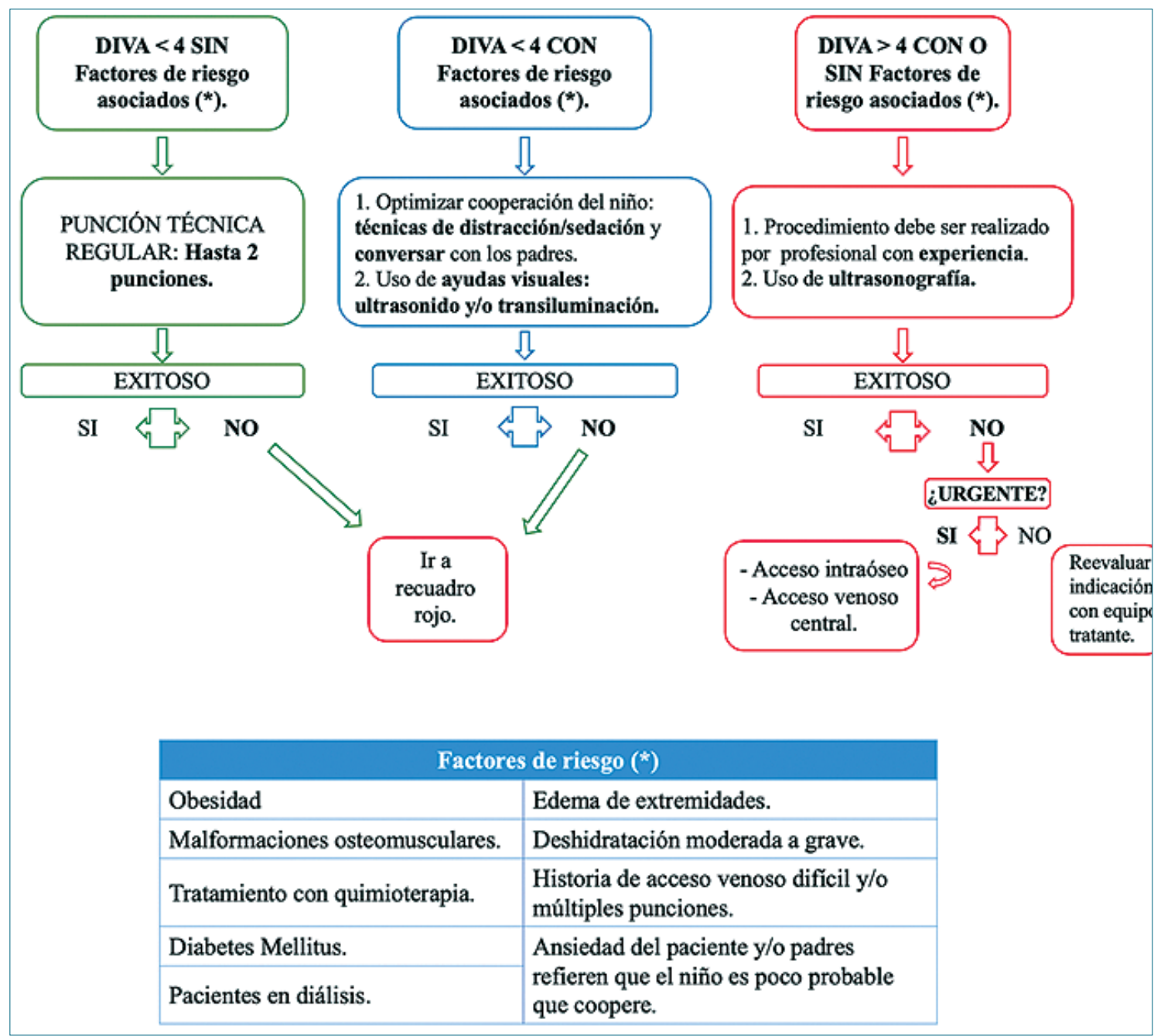

Figura 2. Algoritmo de trabajo. DIVA: DIVA Score de acceso venoso difícil. Se sugiere utilizar alguna técnica de sedación cuando se requiere acceder a un acceso intraóseo o un catéter venoso central.

Recientemente, se han implementado nuevas técnicas para la visualización de los trayectos venosos que utilizan ondas en el espectro cercano al infrarrojo para localizar el flujo de sangre venosa, proyectando luego una imagen virtual del vaso sanguíneo sobre la piel o el sitio anatómico donde se va a realizar la punción. En otras palabras, estos sistemas dibujan una plantilla sobre la superficie a puncionar resaltando en un color diferente el tracto venoso por donde se debe realizar la punción[4].

- Requerimiento de técnicas de distracción y/o sedación para aumentar la tasa de éxito: Los pacientes que ingresan para procedimientos ambulatorios y que se encuentran ansiosos se benefician de la sedación previa al procedimiento, o bien, iniciar el procedimiento con una inducción anestésica inhalatoria y luego la canulación de la vena, lo que permite una adecuada inmovilización de la extremidad y mejores condiciones para la canulación.

- Utilidad de aplicar crema tópica de anestésico local (EMLA o similar).

Por último, se debe seguir insistiendo que el ayuno previo a una cirugía electiva no es sinónimo de necesidad de obtención de una vía venosa periférica, como aún sucede frecuentemente. Con las recomendaciones de ayuno actuales, los pacientes pediátricos pueden recibir líquidos claros por vía oral hasta dos horas antes del procedimiento, por lo que la instalación de una vía venosa para hidratación preoperatoria no es necesaria.

En resumen, el flujo de trabajo se puede sistematizar en el algoritmo que se describe en la Figura 2.

\section{Conclusiones}

En el momento de tomar la decisión de obtener un acceso venoso se deben tener en cuenta los criterios y factores de riesgo de acceso venoso difícil. La escala más reconocida hasta el momento para predecir un acceso venoso difícil es el DIVA Score. Un paciente con más de 4 puntos en la escala se considera paciente con acceso venoso difícil. Sin embargo, la escala no contempla otras variables que también se han asociado a acceso venoso difícil. El uso del ultrasonido y la transiluminación son herramientas que permiten mejorar la tasa de éxito en 
la canulación de estos pacientes. El ayuno preoperatorio no es indicación de instalación de una vía venosa.

\section{Referencias}

1. Kleidon, TM, Cattanach, P, Mihala, G, Ullman, AJ. Implementation of a paediatric peripheral intravenous catheter care bundle: A quality improvement initiative. J Paediatr Child Health 2019; 55 : 1214-23.

2. Schults, J, Rickard, C, Kleidon, T, Paterson, R, Macfarlane, F, Ullman, A. Difficult Peripheral Venous Access in Children: An International Survey and Critical Appraisal of Assessment Tools and Escalation Pathways. J Nurs Scholarsh 2019; 51: 537-46.

3. Girotto, C, Arpone, M, Frigo, AC, Micheletto, M, Mazza, A, Da Dalt, L, y cols. External validation of the DIVA and DIVA3 clinical predictive rules to identify difficult intravenous access in paediatric patients. Emerg Med J 2020; 37: 762-67.

4. Gaurín, C, Acceso Venoso Difícil en Pediatría. 2014 (última actualización: 19-03-2014). Fundación HOMI - Hospital Pediátrico de la Misericordia. Bogotá, C editors. https://homianestesiapediatrica.wordpress.com/2014/03/19/acceso-venoso-dificil-en-pediatria/ 12-04-21 (fecha de acceso).

5. Davis, EM, Feinsmith, S, Amick, AE, Sell, J, McDonald, V, Trinquero, $P, y$ cols. Difficult intravenous access in the emergency de- partment: Performance and impact of ultrasound-guided IV insertion performed by nurses. Am J Emerg Med 2020.

6. Munshey, F, Parra, DA, McDonnell, C, Matava, C. Ultrasoundguided techniques for peripheral intravenous placement in children with difficult venous access. Paediatr Anaesth 2020; 30: 108-15.

7. Riker, MW, Kennedy, C, Winfrey, BS, Yen, K, Dowd, MD. Validation and refinement of the difficult intravenous access score: a clinical prediction rule for identifying children with difficult intravenous access. Acad Emerg Med 2011; 18: 1129-34.

8. Loon, F, Puijn, L, Houterman, S, Bouwman, ARA. Development of the A-DIVA Scale: A Clinical Predictive Scale to Identify Difficult Intravenous Access in Adult Patients Based on Clinical Observations. Medicine (Baltimore) 2016; 95: e3428.

9. Carr, PJ, Rippey, JC, Cooke, ML, Bharat, C, Murray, K, Higgins, NS, $y$ cols. Development of a clinical prediction rule to improve peripheral intravenous cannulae first attempt success in the emergency department and reduce post insertion failure rates: the Vascular Access Decisions in the Emergency Room (VADER) study protocol. BMJ Open 2016; 6: e009196.

10. Shokoohi, H, Loesche, MA, Duggan, NM, Liteplo, AS, Huang, C, Al Saud, AA, y cols. Difficult intravenous access as an independent predictor of delayed care and prolonged length of stay in the emergency department. J Am Coll Emerg Physicians Open 2020; 1: $1660-68$. 